\title{
Reliability Properties of Extended Makeham Distributions
}

\author{
A. H. EL-Bassiouny*, N. F. Abdo \\ Mathematics Department \\ Faculty of Science, Mansoura University, Mansoura, Egypt \\ *e-mail: bassiouny@mans.edu.eg
}

(Received: 23 January 2009; revised: 15 June 2009; accepted: 17 July 2009; published online: 2 October 2009)

\begin{abstract}
This paper investigates reliability properties of a flexible extended Makeham family of distributions. Quantiles are obtained and a probability distribution has for particular parameter values a bathtub hazard rate function.
\end{abstract}

Key words: Makeham distributions, reliability function, moments and quantiles

\section{Symbols}

EM - Extended Makeham

RF - Reliability Function

RV - Random Variable

pdf - probability density function

cdf - cumulative distribution function

FR - Failure Rate

\section{INTRODUCTION}

The Makeham distribution describes the age dynamics of human mortality rather accurately in the age window of about 30-80 years. At more advanced ages the death rates do not increase as fast as predicted by this mortality law - a phenomenon known as the late-life mortality deceleration. Historical decline in human mortality before $1950 \mathrm{~s}$ was mostly due to the decrease in the age-independent mortality component (Makeham parameter), while the agedependent mortality component was surprisingly stable in history before 1950s. After that a new mortality trend has started, leading to unexpected decline in mortality rates at advanced ages and "de-rectangularization" of the survival curve.

In terms of the reliability theory, the Makeham distribution of mortality represents a failure law, where the hazard rate is a mixture of non-aging failure distribution, and the aging failure distribution with exponential increase in failure rates (see [7]).

A new probability distribution which for particular parameter values (three parameters) has a bimodal density function and a bathtub hazard rate function is presented in [3]. Feng et al. [2] use the least squares type estimation to estimate the parameters of Makeham distribution (three parameters).

Adding parameters to a well-established family of distributions is a time-honoured device for obtaining more flexible new families of distributions. An ingeneous general method of adding a parameter to a family of distributions is introduced in [5]. Their method: if we have a family of distributions with $\mathrm{RF} \bar{F}_{0}(x)$, then a new family of distributions can be constructed based on the relation:

$$
\begin{gathered}
\bar{F}(x)=\frac{\gamma \bar{F}_{0}(x)}{1-\bar{\gamma} \bar{F}_{0}(x)}, \\
-\infty<x<\infty, \quad \gamma>0, \quad \bar{\gamma}=1-\gamma .
\end{gathered}
$$

They discovered that the method has a stability property, namely, if the method is applied twice, nothing new will be obtained the second time around. They also have shown that the newly generated family of distributions with the RF (1) is geometric extreme stable, namely, if $X_{1}, X_{2}, \ldots$ is a sequence of independent and identically distributed RVs with RF (1), independent of a RV $N$ possessing a geometric distribution with the probability mass function:

$$
\begin{aligned}
& P(N=n)=p(1-p)^{n-1}, \\
& n=1,2, \ldots, \quad 0<p<1,
\end{aligned}
$$

then the RVs 


$$
\begin{aligned}
& U=\min \left(X_{1}, X_{2}, \ldots, X_{N}\right), \\
& V=\max \left(X_{1}, X_{2}, \ldots, X_{N}\right),
\end{aligned}
$$

also belong to the family of distributions with the RF (1).

In this paper we introduce a new variant of MarshallOlkin extended family of distributions by selecting in (1) the RF of Makeham distribution with three parameters:

$$
\begin{gathered}
\bar{F}_{0}(x)=\exp \left(-A x-\frac{B}{\alpha}\left(e^{\alpha x}-1\right)\right), \\
x>0, \quad A, B, \alpha>0 .
\end{gathered}
$$

Substituting (4) in (1), we obtain

$$
\begin{gathered}
\bar{F}(x)=\frac{\gamma \exp \left(-A x-\frac{B}{\alpha}\left(e^{\alpha x}-1\right)\right)}{1-\bar{\gamma} \exp \left(-A x-\frac{B}{\alpha}\left(e^{\alpha x}-1\right)\right)}= \\
=\frac{\gamma}{\exp \left(A x+\frac{B}{\alpha}\left(e^{\alpha x}-1\right)\right)-\bar{\gamma}}, \\
x>0, \quad A, B, \alpha, \gamma>0 .
\end{gathered}
$$

We shall write $X \sim E M(A, B, \alpha, \gamma)$ to denote an absolutely continuous RV $X$ possessing the Marshall-Olkin extended Makeham distribution with parameters $A, B, \alpha, \gamma$ and $\mathrm{RF}$ given by (5).

The aim of this paper is to reveal some reliability properties of $E M$ distribution. These properties include: (i) expressing this proposed distribution as a compounding process with exponential mixing model, (ii) shapes of the probability density function, (iii) moments and quantiles, (iv) shapes of the failure rate function, (v) stochastic ordering, and (vi) limiting distributions of extreme order statistics.

\section{COMPOUNDING}

Let $\bar{F}(x \mid \theta),-\infty<x<\infty,-\infty<\theta<\infty$, be the conditional RF of a continuous RV $X$ given a continuous RV $\Theta$. Let $\Theta$ follow a distribution with the pdf $m(\theta)$. A distribution with RF

$$
\begin{gathered}
\bar{F}(x)=\int_{-\infty}^{\infty} \bar{F}(x \mid \theta) m(\theta) d \theta, \\
-\infty<x<\infty
\end{gathered}
$$

is called a compound distribution with mixing density $m(\theta)$. Compound distribution provides a tool for obtaining new parametric families of distributions in terms of existing ones. They represent heterogeneous models where populations items involve different risks.

The following theorem shows that the EM distribution can be expressed by the compounding argument.

\section{THEOREM 1}

Let the conditional RF of a continuous RV $X$ given $\Theta=\theta$ be expressed as:

$$
\bar{F}(x)=\exp \left(-\left(e^{A x+\frac{B}{\alpha}\left(e^{\alpha x}-1\right)}-1\right) \theta\right)
$$

$$
x, \theta>0, \quad A, B, \alpha>0 .
$$

Let $\Theta$ follows an exponential distribution with probability density function (pdf):

$$
m(\theta)=\gamma e^{-\gamma \theta}, \quad \theta>0, \quad \gamma>0 .
$$

Then the compound distribution of $X$ is the $\operatorname{EM}(A, B, \alpha, \gamma)$ distribution.

Proof.

For all $x>0, A, B, \alpha, \gamma>0$ the unconditional RF of $X$ is given by

$$
\begin{gathered}
\bar{F}(x)=\int_{0}^{\infty} \bar{F}(x \mid \theta) m(\theta) d \theta= \\
=\gamma \int_{0}^{\infty} \exp \left(-\left(e^{A x+\frac{B}{\alpha}\left(e^{\alpha x}-1\right)}-\bar{\gamma}\right) \theta\right) d \theta= \\
=\frac{\gamma}{\exp \left(A x+\frac{B}{\alpha}\left(e^{\alpha x}-1\right)\right)-\bar{\gamma}},
\end{gathered}
$$

which is indeed the RF (5) of $E M(A, B, \alpha, \gamma)$ distribution.

\section{THE PROBABILITY DENSITY FUNCTION}

The pdf of the $\operatorname{EM}(A, B, \alpha, \gamma)$ distribution with RF (5), is given by

$f(x)=-\frac{d \bar{F}(x)}{d x}=\frac{\gamma\left(A+B e^{\alpha x}\right) \exp \left(A x+\frac{B}{\alpha}\left(e^{\alpha x}-1\right)\right)}{\left(\exp \left(A x+\frac{B}{\alpha}\left(e^{\alpha x}-1\right)\right)-\bar{\gamma}\right)^{2}}$,

$$
x>0, A, B, \alpha, \gamma>0 .
$$


The following theorem gives simple conditions under which the pdf (10) is decreasing or unimodal.

\section{THEOREM 2}

Let $X \sim E M(A, B, \alpha, \gamma)$ then $X$ has a decreasing (unimodal) pdf provided $(\gamma-2)\left(A^{2}+2 A B+B^{2}\right)+\alpha B \gamma \leq 0(>0)$.

Proof.

The first derivative of $f(x)$ is given by

$$
f^{\prime}(x)=\frac{\exp \left(A x+\frac{B}{\alpha}\left(e^{\alpha x}-1\right)\right) \psi(x)}{\left(\exp \left(A x+\frac{B}{\alpha}\left(e^{\alpha x}-1\right)\right)-\bar{\gamma}\right)^{3}}, \quad x>0
$$

where

$$
\begin{aligned}
\psi(x)= & \left(B e^{\alpha x}(-2 A+\alpha)-A^{2}-B^{2} e^{2 \alpha x}\right) e^{A x+\frac{B}{\alpha}\left(e^{\alpha x}-1\right)}+ \\
& -\bar{\gamma}\left(B e^{\alpha x}(2 A+\alpha)+A^{2}+B^{2} e^{2 \alpha x}\right)
\end{aligned}
$$

The function $\psi(x)$ has no (one) zero on $(0, \infty)$ provided $\psi(0)=(\gamma-2)\left(A^{2}+2 A B+B^{2}\right)+\alpha B \gamma \leq 0(>0)$. That is, $f(x)$ has no (one) critical point provided $\psi(0) \leq 0(>0)$. Since $f(x)$ is non-negative and $f(0)=(A+B) / \gamma$, $f(\infty)=0$ then $f(x)$ is decreasing (unimodal) provided $\psi(0) \leq 0(>0)$.

\section{Remarks:}

(i) When $\gamma \in[2, \infty), f(x)$ is unimodal for all values of $A, B, \alpha>0$. Otherwise, $f(x)$ may still exhibit a decreasing or unimodal behavior according to the conditions of theorem 2 .

(ii) As $B \rightarrow 0$, i. e. the extended exponential distribution, $f(x)$ is decreasing (unimodal) provided $0<\gamma \leq 2(\gamma>2)$, as shown by [5].

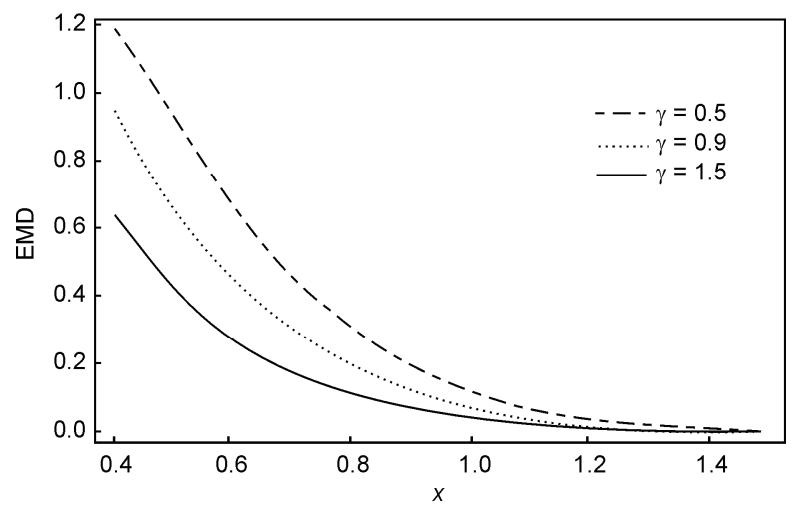

Fig. 1. Plot of the pdf fordifferent values of $\gamma$ for $A=\alpha=1, B=2$

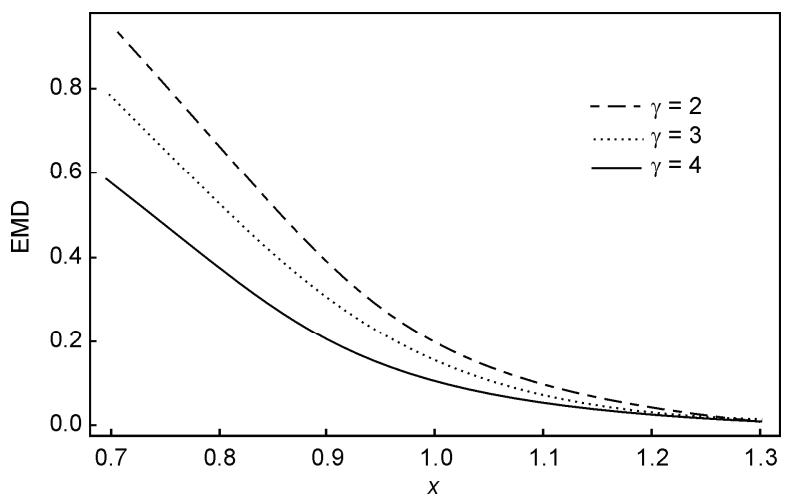

Fig. 2. Plot of the pdf for different values of $\gamma$ for $A=\alpha=2, B=1$

\section{MOMENTS AND QUANTILES}

The $r t h$ moment of the $E M$ distribution is given by

$$
\begin{gathered}
E\left(X^{r}\right)=r \int_{0}^{\infty} x^{r-1} \bar{F}(x) d x= \\
=r \gamma \int_{0}^{\infty} \frac{x^{r-1}}{\exp \left(A x+\frac{B}{\alpha}\left(e^{\alpha x}-1\right)\right)-\bar{\gamma}} d x, \\
r=1,2, \ldots
\end{gathered}
$$

In general, the last integral cannot be given explicitly in terms of $r, \alpha, \gamma, A, B$. The mean $E(X)$ and the variance $\operatorname{Var}(\mathrm{X})$ of $E M$ distribution are shown graphically in the following Figures for $A=1, \alpha=3, B=2$ :

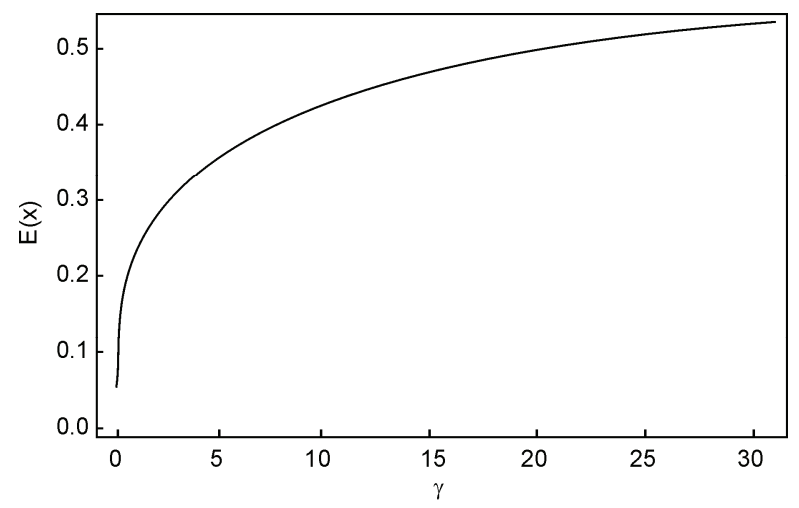

Fig. 3. Plot of the mean 


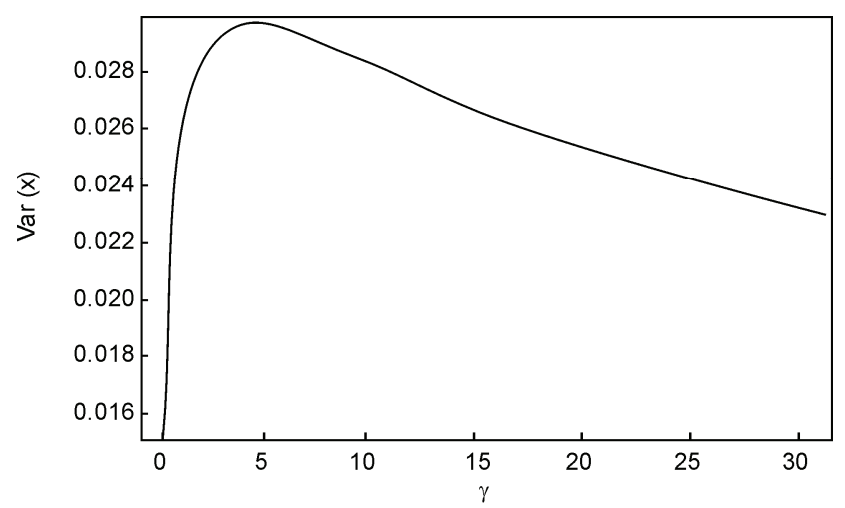

Fig. 4. Plot of the variance

The qth quantile of the $E M$ is given by

$$
\begin{gathered}
x_{q}=F^{-1}(q)= \\
=\frac{-1}{A \alpha}\left(\text { Lambert } W\left(\frac{B}{A} e^{\frac{1}{A}\left(B+\alpha \ln \left(\frac{1-q+q \gamma}{1-q}\right)\right)}\right) A+\right. \\
\left.-B-\alpha \ln \left(\frac{1-q+q \gamma}{1-q}\right)\right)
\end{gathered}
$$

where $F^{-1}($.$) is the inverse distribution function.$

The median of the $E M$ distribution, i. e. $x_{0.5}$, is given by

$$
\begin{gathered}
\text { median }(X)= \\
=\frac{-1}{A \alpha}\left(\text { Lambert } W\left(\frac{B}{A} e^{\frac{1}{A}(B+\alpha \ln (1+\gamma))}\right) A+\right. \\
-B-\alpha \ln (1+\gamma)) .
\end{gathered}
$$

\section{FAILURE RATE FUNCTION}

The concept of the FR in engineering (also known as the hazard rate in medical science and the force of mortality in actuarial science) is the basic staple of the modern reliability theory.

The FR function of the $\operatorname{EM}(A, B, \alpha, \gamma)$ distribution is given by

$$
\begin{gathered}
h(x)=\frac{f(x)}{\bar{F}(x)}= \\
=\frac{\exp \left(A x+\frac{B}{\alpha}\left(e^{\alpha x}-1\right)\right)\left(A+B e^{x \alpha}\right)}{\exp \left(A x+\frac{B}{\alpha}\left(e^{\alpha x}-1\right)\right)-\bar{\gamma}} .
\end{gathered}
$$

and FR average function of the $\operatorname{EM}(A, B, \alpha, \gamma)$ distribution is given by

$$
h_{s}(x)=\frac{1}{x} \int_{0}^{x} h(u) d u=
$$

$$
=\frac{\log \left(\exp \left(A x+\frac{B}{\alpha}\left(e^{\alpha x}-1\right)\right)-\bar{\gamma}\right)-\log (\gamma)}{x} .
$$

The behavior of the FR and FR average is the same. The following remark presents a formal description of the possible shapes of the FR of the $E M$ distribution in terms of the parameters $A, B, \alpha, \gamma$.

\section{THEOREM 3}

Let

$\xi(x)=B e^{\frac{B\left(-1+e^{x \alpha}\right)}{\alpha}+A x+x \alpha} \alpha-\bar{\gamma}\left(A^{2}+B^{2} e^{2 x \alpha}+B e^{x \alpha}(2 A+\alpha)\right)$, $x>0$. If $X \sim \operatorname{EM}(A, B, \alpha, \gamma)$, then $X$ has

(i) a decreasing FR provided

$$
0<\gamma<0.1, \quad \xi(0)<0
$$

(ii) a decreasing-increasing (bathtub) FR provided

$$
0.1<\gamma<0.5, \quad \xi(0)<0
$$

(iii) an increasing FR provided

$$
\gamma>0.5, \quad \xi(0)>0 .
$$

Proof.

The first derivative of $h(x)$ with respect to $x$ is given by

$$
h^{\prime}(x)=\frac{e^{\frac{B\left(-1+e^{x \alpha}\right)}{\alpha}+A x} \xi(x)}{\left(\gamma+e^{\frac{B\left(-1+e^{x \alpha}\right)}{\alpha}+A x}-1\right)^{2}},
$$


where

$$
\begin{gathered}
\xi(x)=B e^{\frac{B\left(-1+e^{x \alpha}\right)}{\alpha}+A x+x \alpha} \alpha+ \\
-\bar{\gamma}\left(A^{2}+B^{2} e^{2 x \alpha}+B e^{x \alpha}(2 A+\alpha)\right) .
\end{gathered}
$$

Note that

$\xi(0)=B \alpha-\bar{\gamma}\left(A^{2}+B^{2}+B(2 A+\alpha)\right)$ and $\xi(\infty)=\infty$.

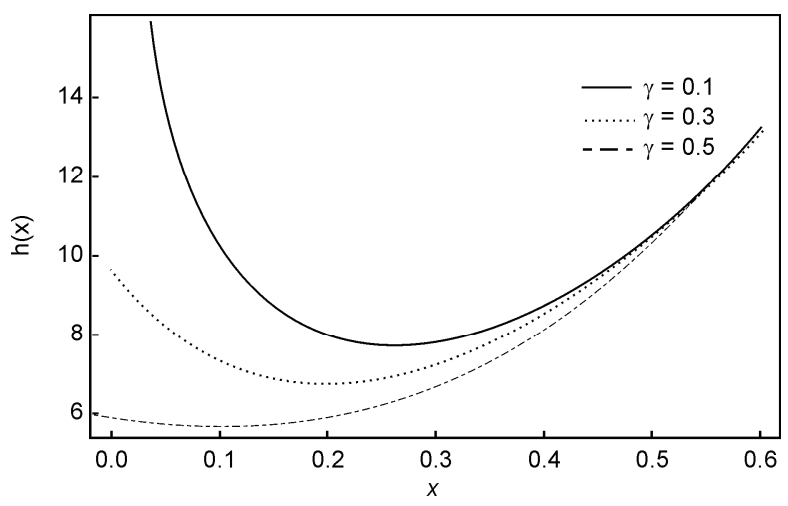

Fig. 5. Plot of the FR for different values of $\gamma$ for $A=1, \alpha=3, B=2$

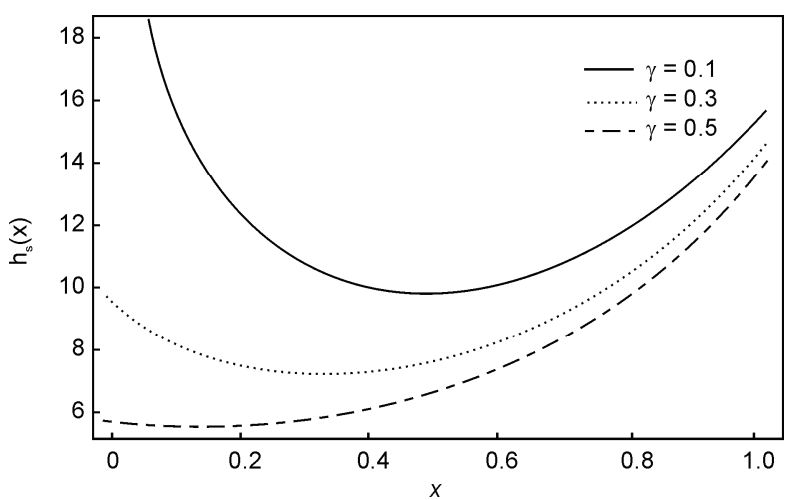

Fig. 6. Plot of the FR average for different values of $\gamma$ for $A=1$, $\alpha=3, B=2$

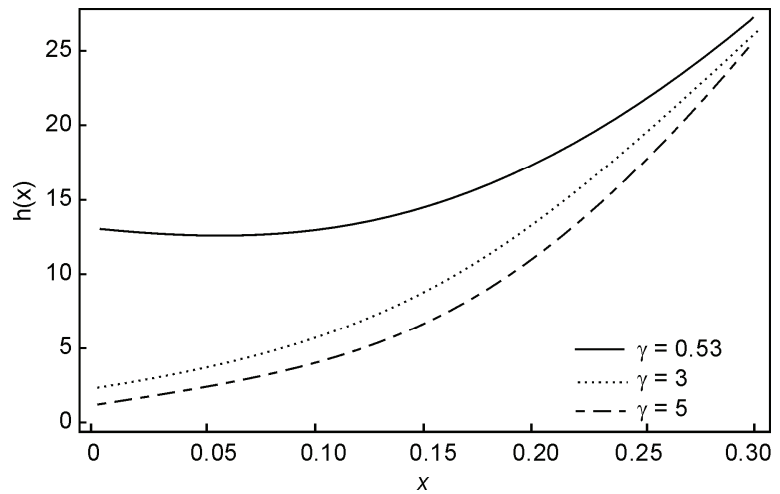

Fig. 7. Plot of the FR for different values of $\gamma$ for $A=3, \alpha=6, B=4$

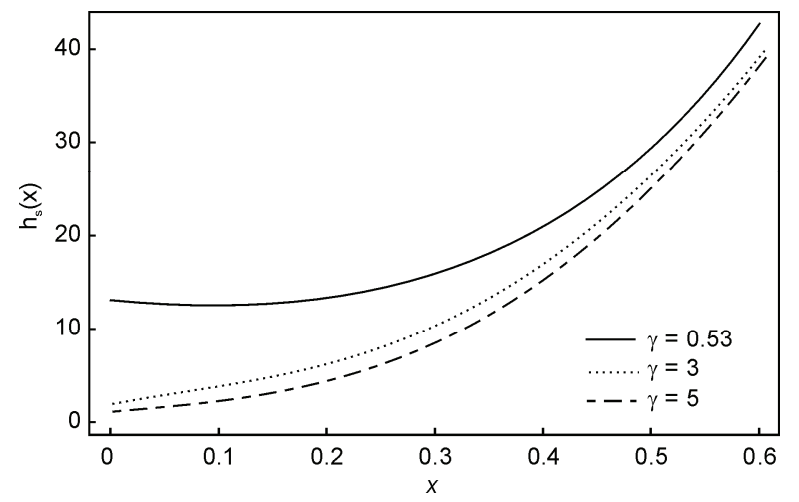

Fig. 8. Plot of the FR average for different values of $\gamma$ for $A=3$, $\alpha=6, B=4$

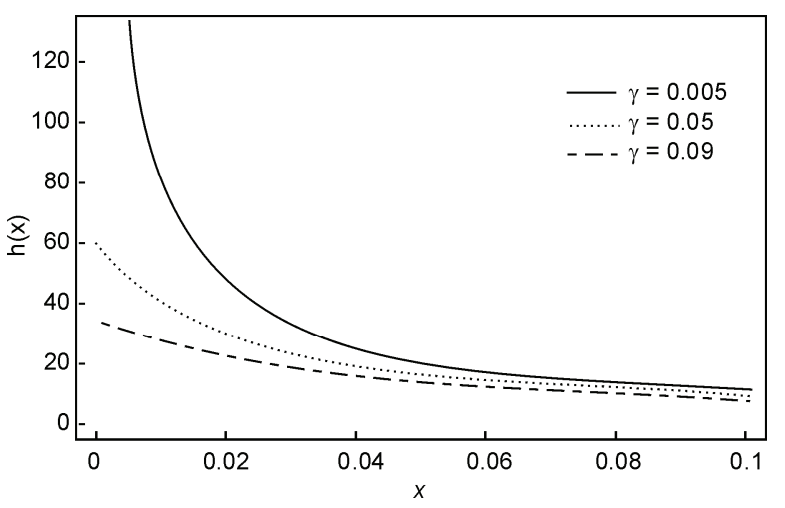

Fig. 9. Plot of the FR for different values of $\gamma$ for $A=3$, $\alpha=2.6, B=0.25$

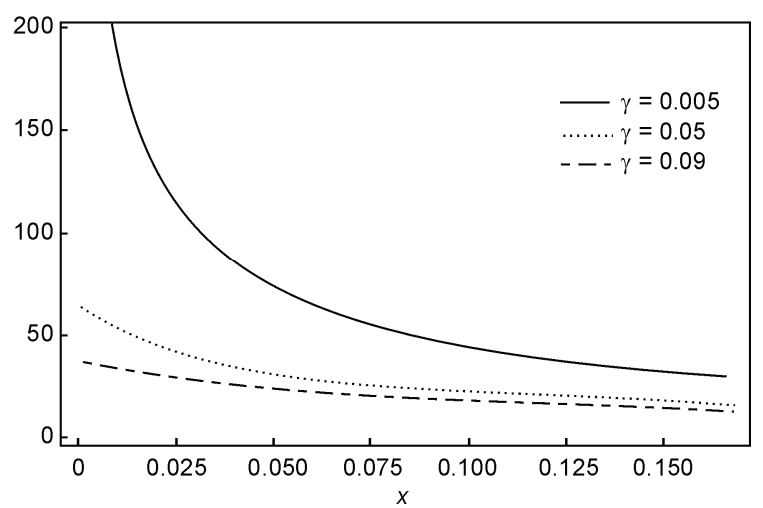

Fig. 10. Plot of the FR average for different values of $\gamma$ for $A=3$, $\alpha=2.6, B=0.25$

\section{STOCHASTIC ORDERINGS}

Stochastic ordering of positive continuous random variables is an important tool to judge the comparative behavior. We shall recall some basic definitions. 
Random variable $X$ is said to be smaller than random variable $Y$ in the

(i) stochastic order (denoted by $X \leq_{s t} Y$ ) if $\bar{F}_{X}(x) \leq \bar{F}_{Y}(x)$ for all $x$;

(ii) hazard rate order (denoted by $X \leq_{h r} Y$ ) if $h_{X}(x) \geq h_{Y}(x)$ for all $x$

(iii) likelihood ratio order (denoted by $X \leq_{l r} Y$ ) if $f_{X}(x) / f_{Y}(x)$ decreases in $x$.

The following implications (see [6] chapter 9) are well known:

$$
X \leq_{l r} Y \Rightarrow X \leq_{h r} Y \Rightarrow X \leq_{s t} Y .
$$

The EM distributions are ordered with respect to the strongest "likelihood ratio" ordering as shown in the following theorem.

\section{THEOREM 4}

Let

$$
\begin{gathered}
X \sim \operatorname{EM}\left(A, B, \alpha, \gamma_{1}\right) \\
\text { and } Y \sim E M\left(A, B, \alpha, \gamma_{2}\right) .
\end{gathered}
$$

If $\gamma_{1}<\gamma_{2}$, then

$$
X \leq_{l r} Y\left(X \leq_{h r} Y, X \leq_{s t} Y\right)
$$

Proof.

First note that

$$
\begin{gathered}
\frac{f_{X}(x)}{f_{Y}(x)}= \\
=\frac{\gamma_{1}}{\gamma_{2}}\left[\frac{\exp \left(A x+\frac{B}{\alpha}\left(e^{\alpha x}-1\right)\right)-\bar{\gamma}_{2}}{\exp \left(A x+\frac{B}{\alpha}\left(e^{\alpha x}-1\right)\right)-\bar{\gamma}_{1}}\right]^{2}
\end{gathered}
$$

Since, for $\gamma_{1}<\gamma_{2}$,

$$
\begin{gathered}
\frac{d}{d x} \frac{f_{X}(x)}{f_{Y}(x)}=2 \frac{\gamma_{1}}{\gamma_{2}}\left(\gamma_{1}-\gamma_{2}\right) \times \\
\times \frac{\left(\exp \left(A x+\frac{B}{\alpha}\left(e^{\alpha x}-1\right)\right)-\bar{\gamma}_{2}\right)}{\left(\exp \left(A x+\frac{B}{\alpha}\left(e^{\alpha x}-1\right)\right)-\bar{\gamma}_{1}\right)^{3}} \times \\
\times\left(A+B e^{\alpha x}\right) e^{A x+\frac{B}{\alpha}\left(e^{\alpha x}-1\right)}<0,
\end{gathered}
$$

then $f_{X}(x) / f_{Y}(x)$ is decreasing in $x$. That is $X \leq_{l r} Y$. The remaining statements follow from the implications (19).

\section{LIMITING DISTRIBUTIONS OF SAMPLE EXTREMES}

Let $X_{1}, X_{2}, \ldots, X_{n}$ be a random sample of size $\mathrm{n}$ from an absolutely continuous distribution with pdf $f(x)$ and cdf $F(x)=1-\bar{F}(x)$. Limiting distribution of sample maxima $X_{n: n}=\max \left(X_{1}, X_{2}, \ldots, X_{n}\right)$ is a long stand area in applications of probability and statistics (see, e.g. [4]). First, we recall the following asymptotical results for $X_{n: n}$ (see, e.g., [1], pp. 210-214).

(i) For the maximal order statistic $X_{n: n}$, we have

$$
\lim _{n \rightarrow \infty} P\left\{X_{n: n} \leq a_{n}+b_{n} t\right\}=\exp \left(-e^{-t}\right),
$$

$$
-\infty<t<\infty,
$$

(of the extreme value type) where

$$
a_{n}=F^{-1}\left(1-\frac{1}{n}\right)
$$

$$
\text { and } b_{n}=\frac{1}{n f\left(a_{n}\right)} \text { if }
$$

$$
\lim _{x \rightarrow \infty} \frac{d}{d x}\left(\frac{1}{h(x)}\right)=0
$$

The following theorem derives the limiting distributions of the largest order statistics from the $E M$ distribution.

\section{THEOREM 5}

Let $X_{n: n}$ be the largest order statistics from $\operatorname{EM}(A, B$, $\alpha, \gamma)$ distribution. Then

$$
\begin{gathered}
\lim _{n \rightarrow \infty} P\left\{X_{n: n} \leq a_{n}+b_{n} t\right\}=\exp \left(-e^{-t}\right), \\
-\infty<t<\infty
\end{gathered}
$$

where

$$
a_{n}=F^{-1}\left(1-\frac{1}{n}\right) \text { and } b_{n}=\frac{1}{n f\left(a_{n}\right)}
$$

(22) and $f(),. F^{-1}($.$) , respectively, are given by (10) and (14).$

Proof.

For $E M$ distribution, we have 


$$
\lim _{x \rightarrow \infty} \frac{d}{d x}\left(\frac{1}{h(x)}\right)=\lim _{x \rightarrow \infty} \frac{e^{\frac{-e^{x \alpha} B+B-A x \alpha}{\alpha}}\left(-B e^{\frac{B\left(-1+e^{x \alpha}\right)}{\alpha}+x(A+\alpha)} \alpha-\left(A^{2}+B^{2} e^{2 x \alpha}+B e^{x \alpha}(2 A+\alpha)\right)(\gamma-1)\right)}{\left(A+B e^{x \alpha}\right)^{2}}=0 .
$$

Hence, the statement follows from (23) and (24).

\section{CONCLUSION}

The new extended Makeham distribution is obtained. The proposed extended Makeham distribution has a unimodal probability density function. Such characteristics are useful for modeling continuous data from life-testing experiments. Some reliability properties are obtained. The extended Makeham distributions are ordered with respect to the likelihood ratio stochastic ordering. The limiting distribution of the largest order statistics of a random sample from the proposed distribution has an extreme-value distribution.

The distribution profile is shown in Figs. (1-2). On the other hand, Figs. (5-10) show the variations of FR and FR average for different values of $\gamma$. The behavior of FR and FR average is the same, it is clear that for $0.1 \leq \gamma \leq 0.5$, $\xi(0)<0$, decreasing-increasing (bathtub) FR and FR average are obtained and for $\gamma>0.5, \xi(0)>0$, increasing FR and FR average are obtained, while for $0 \leq \gamma \leq 0.1$, $\xi(0)<0$, decreasing FR and FR average are obtained.

\section{References}

[1] B.C. Arnold, N. Balakrishnan and H.N. Nagaraja, A first course in order statistics. NewYork, Wiley (1992).

[2] X. Feng, G. He and Abdurishit, Estimation of parameters of the Makeham distribution using the least squares method. Mathematics and Computers in Simulation 77, 34-44 (2008).

[3] S. Kosznik-Biernacka, Makeham's generalised distribution. Computational Methods in Science and Technology 13(2), 113-120 (2007).

[4] S. Kotz and S. Nadarajah, Extreme value theory, theory and applications. Singapore, World Scientific Publishing Company (2001).

[5] A.W. Marshall and I. Olkin, A new method for adding a parameter to a family of distributions with application to the exponential and Weibull families. Biometrika 84, 641-652 (1997).

[6] S.M. Ross, Stochastic processes. Second edition. New York, Wiley (1996).

[7] D.P.M. Scollnik, Simulating random variates from Makeham's distribution and from others with exact or nearly Log-Concave densities. Trans. Soc. Actuaries 47, 409-437 (1995).

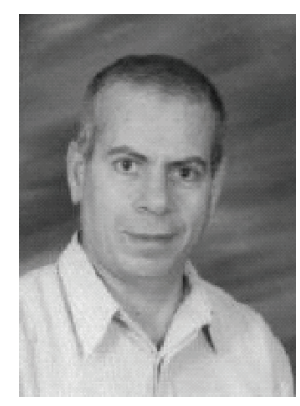

Ahmed Habib El-Bassiouny. Professor of Mathematical Statistics, Head of Mathematics Department, Faculty of Science, Mansoura University, Mansoura, Egypt. Graduated in mathematics in 1977. Since then he has been working in Mansoura University. He earned the Ph.D. in probability and statistics in 1986 from Kiev State University, Ukraine, U.S.S.R. His research interests concern mathematical and computational methods in statistics.

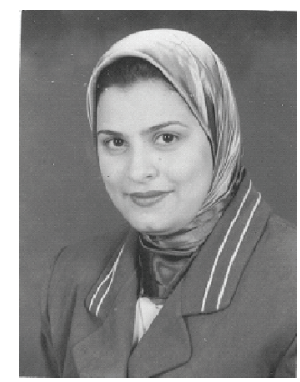

Noura Fakhry Abdo Abd El Latef. Ph.D. student, graduated in mathematics in 1999. Since then she has been with the Mathematics Department, Faculty of Science, Mansoura University, Mansoura, Egypt. In 2006, she earned the master's degree in statistics and computer science from Mansoura University. Her research interests concern mathematical and computational methods in statistics. 